\title{
Understanding glucose via postprandial plasma glucose sensor data segmentation analysis (GH-Method: math-physical medicine)
}

Gerald C. Hsu

EclaireMD Foundation, USA

\section{Introduction}

In this paper, the author analyzed and interpreted a type 2 diabetes (T2D) patient's postprandial plasma glucose (PPG) waveforms (i.e. curve sets), data, and key characteristics, with mathematical tools to study the physical behaviors of glucose in detail, utilizing his developed GH-Method: math-physical medicine approach.

\section{Methods}

The author was diagnosed with T2D 25 years ago. He has collected his blood sugar values via a continuous glucose monitoring device (the Sensor) that was applied to his upper arm during the period of 5/5/2018 - 9/28/2019. As shown in Figure 1, during these 481 days, he has collected a total of 35,748 glucose data (total 100\%) with an averaged 74 collections per day. His big glucose data included 481 fasting plasma glucose (FPG) waveforms with 5,291 data $(15 \%$ of total), 1,443 PPG waveforms with 18,759 data (52\% of total), and 11,698 pre-period glucose data (both pre-meals and prebed for $33 \%$ of total).

After collecting sufficient data and also segregating them into proper categories for analysis, he initially calculated and plotted out FPG waveform ( $25 \%$ contribution to HbA1C), similar to a salad bowl shape, and PPG waveform ( $\sim 75 \%$ contribution to $\mathrm{HbA} 1 \mathrm{C})$, similar to a mountain shape. Secondly, he calculated the average PPG values at four-time instants, i.e. at 0,60 , 120, 180 minutes, and during three sub-periods, i.e. for $0-60$, 60-120, 60-180 minutes time periods. Thirdly, by applying his developed OHCA (Open-High-Close-Average) model and three decomposed PPG waveform models, known as the Himalaya, Twin Peak, \& Grand Canyon, he calculated their respective average and peak glucoses.

\begin{tabular}{|c|r|r|}
\hline From: 5/5/2018 & & \\
\hline To: 9/28/2019 & & \\
\hline Glucose Type & Sensor & Finger \\
\hline Days & $\mathbf{4 8 1}$ & $\mathbf{4 8 1}$ \\
\hline FPG / Day & $\mathbf{1 1}$ & $\mathbf{1}$ \\
\hline PPG / Day & $\mathbf{3 9}$ & $\mathbf{3}$ \\
\hline Pre- / Day & $\mathbf{2 4}$ & $\mathbf{0}$ \\
\hline Data / Day & $\mathbf{7 4}$ & $\mathbf{4}$ \\
\hline Total Data & $\mathbf{3 5 , 7 4 8}$ & $\mathbf{1 , 9 2 4}$ \\
\hline FPG Data & $\mathbf{5 , 2 9 1}$ & $\mathbf{4 8 1}$ \\
\hline PPG Data & $\mathbf{1 8 , 7 5 9}$ & $\mathbf{1 , 4 4 3}$ \\
\hline Pre- Data & $\mathbf{1 1 , 6 9 8}$ & - \\
\hline FPG Waveforms & $\mathbf{4 8 1}$ & $\mathbf{0}$ \\
\hline PPG Waveforms & $\mathbf{1 4 4 3}$ & $\mathbf{0}$ \\
\hline
\end{tabular}

Figure 1: Data window and Size

\author{
Correspondence author \\ Gerald C Hsu, \\ EclaireMD Foundation \\ USA
}


Results

As shown in Figure 2 and Figure 3, he displayed seven daily PPG curves and their summarized table of average glucoses. Sensor PPG (daily averaged PPG $136 \mathrm{mg} / \mathrm{dL}$ ) rises from a composite opening glucose $128 \mathrm{mg} / \mathrm{dL}$ at the first bite of meal, and then reaches to its composite high peak value of $144 \mathrm{mg} /$ $\mathrm{dL}$ at 60 minutes, and finally decreases to its composite closing $131 \mathrm{mg} / \mathrm{dL}$ at 180 minutes. It should be noted that the PPG's peak occurs approximately 60 minutes after first bite of meal, not the recognized standard of "two hours after your meal".

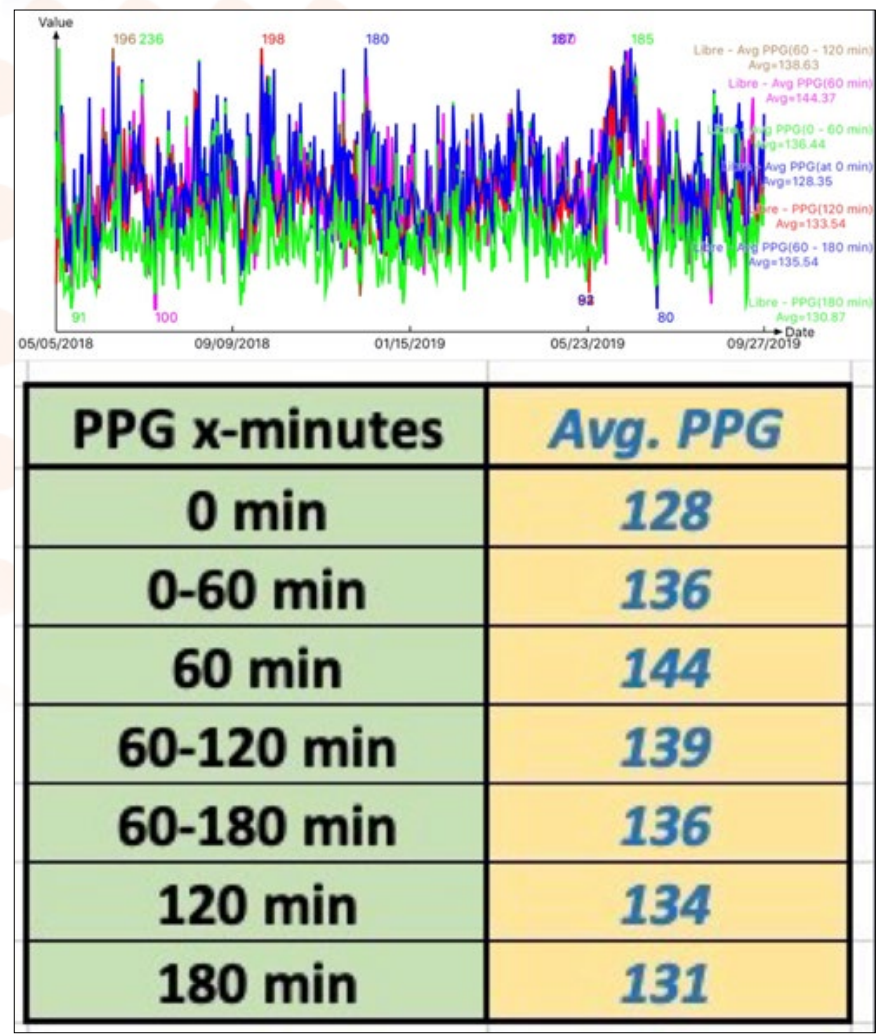

Figure 2: $P P G$ glucose values at different time instants

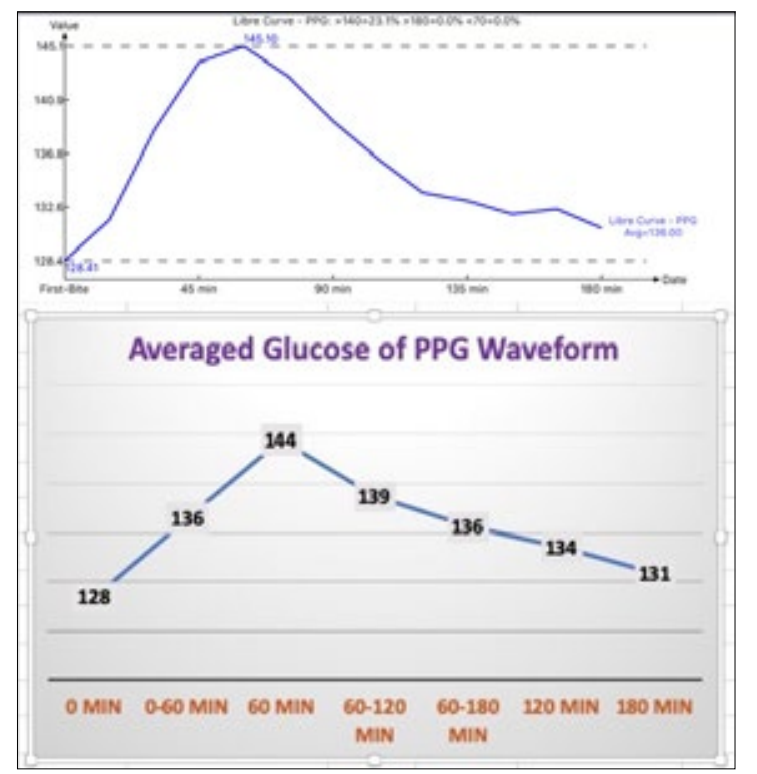

Figure 3: Two PPG waveforms based on overall data composition and assembly from 4 key time instants and 3 subperiods

His OHCA analysis results at three distinctive models: Himalaya (inactivity after meal), Grand Canyon (proper and sufficient post-meal exercise after carbs/sugar intake), and Twin Peaks (insufficient or interrupted post-meal exercise after meal). In Figure 4, by using the Grand Canyon as the $100 \%$ baseline, Twin Peak has $7 \%$ higher average glucose (15\% higher energy) and 4\% higher peak glucose (8\% higher energy), while Himalaya has $1 \%$ higher average glucose (2\% higher energy) and $2 \%$ higher peak glucose $(4 \%$ higher energy). Based on the observation, Himalaya would have the worst PPG results. However, the author possesses ample knowledge of glucose and practical experiences of glucose control; therefore, his intake of carbs/sugar would be less when he anticipated in advance of his upcoming post-meal inactivity situation.

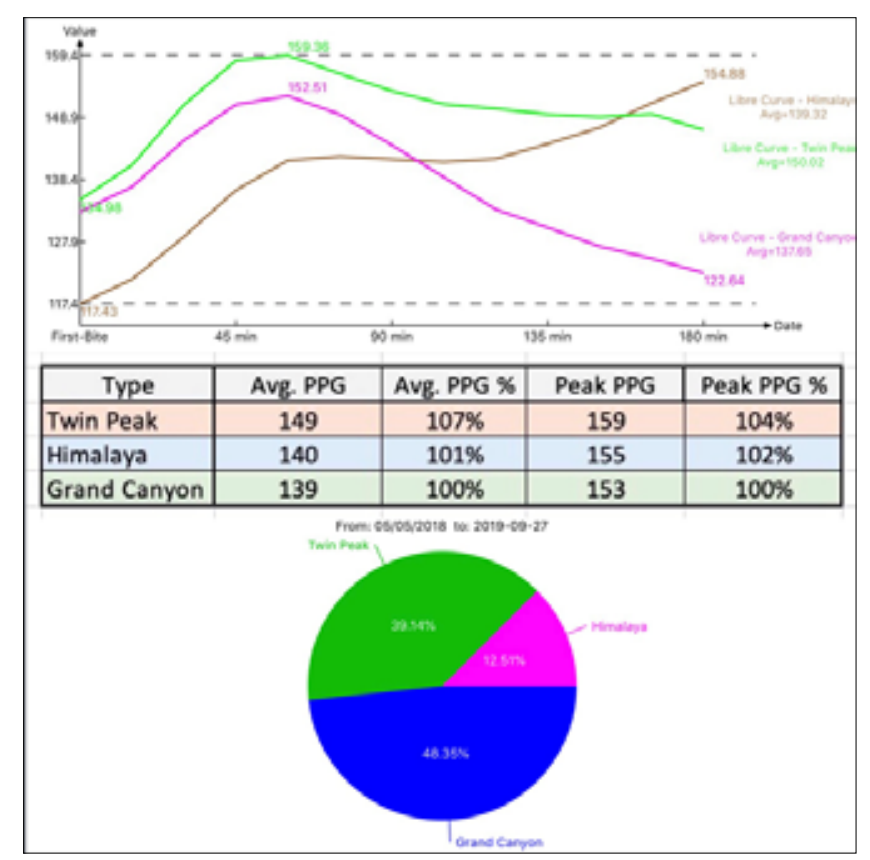

Figure 4: Three standardized sensor PPG waveforms

The high glucose components in his glucose analyses, those with glucose values higher than $140 \mathrm{mg} / \mathrm{dL}$ or $180 \mathrm{mg} / \mathrm{dL}$, would carry excessive leftover energy within the blood which circulates throughout the body and damages the internal organs to create diabetes complications.

Figure 5 shows the rising speed of glucose from 0 minute to 60 minutes and dropping speed of glucose from 60 minutes to 180 minutes. In general, the dropping speed at $19 \mathrm{mg} / \mathrm{dL}$ per hour is at around $60 \%$ level of rising speed at $32 \mathrm{mg} / \mathrm{dL}$ per hour. By knowing your carbs/sugar intake and your starting glucose at 0 minute and then applying the rising speed, you can quickly calculate your peak PPG level around peak time at 60 minutes. Similarly, depending on post-meal exercise pattern and amount, and then applying the proper dropping speed of your exercise amount, you can also calculate your PPG level at 180 minutes after your first bite of meal. The above statements 
describe the exact process of a quantitative PPG analysis.

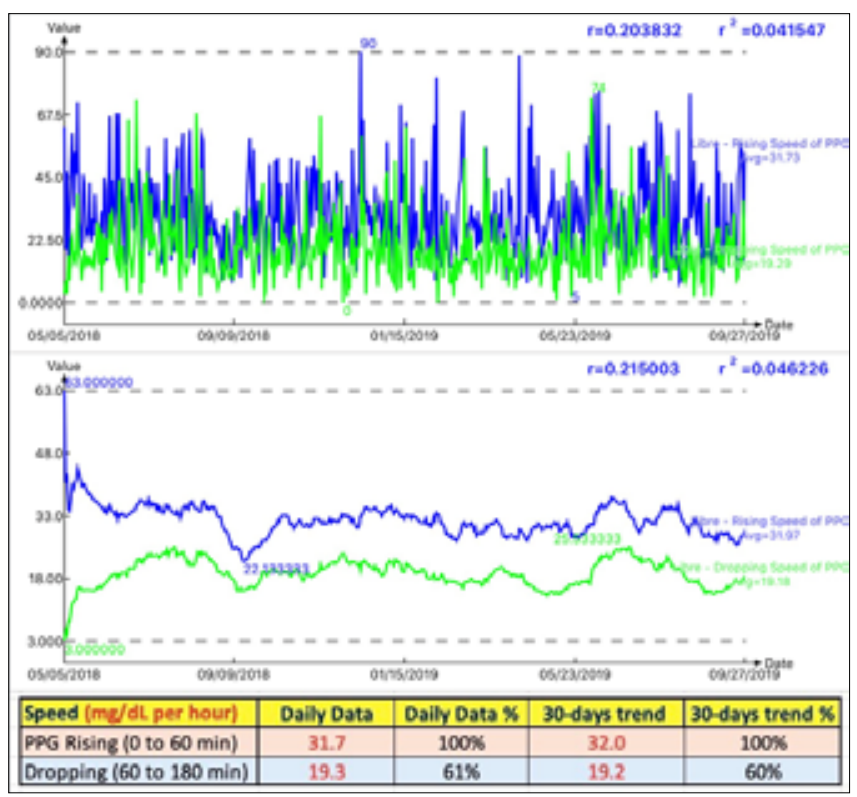

Figure 5: PPG wave's rising speed and dropping speed

\section{Conclusions}

Currently, diabetes is a non-curable chronic disease. There are various medications and insulin injections that can alleviate the symptoms; however, the best option is to control diabetes from its root-cause in order to prevent the horrible complications. For those with T2D who want to control it via an effective lifestyle management program, a thorough and deep understanding of glucose is a prerequisite for healthcare professionals to provide accurate and effective knowledge, advice, and support. Once they fully understand glucose, they are then able to provide useful lifestyle management (food, exercise, and other factors) material and training to diabetes patients. The aim of this paper is to provide the necessary quantitative and qualitative information about the simple term of glucose. If we do not understand Glucose, then how can we claim to understand Diabetes?[1-5].

\section{References}

1. Hsu, Gerald C (2018) Using Math-Physical Medicine to Control T2D via Metabolism Monitoring and Glucose Predictions. Journal of Endocrinology and Diabetes 1(1): 1-6. Retrieved from http://www.kosmospublishers.com/ wp-content/uploads/ 2018/06/JEAD-101-1.pdf

2. Hsu, Gerald C (2018) Using Math-Physical Medicine to Analyze Metabolism and Improve Health Conditions. Video presented at the meeting of the 3rd International Conference on Endocrinology and Metabolic Syndrome 2018, Amsterdam, Netherlands.

3. Hsu, Gerald C (2018) Using Signal Processing Techniques to Predict PPG for T2D. International Journal of Diabetes \& Metabolic Disorders 3(2): 1-3. Retrieved from https:// www.opastonline.com/wp-content/uploads/2018/06/ using-signal-processing-techniques-to-predict-ppg-for- t2d-ijdmd-18.pdf

4. Hsu, Gerald C (2018) Using Math-Physical Medicine and Artificial Intelligence Technology to Manage Lifestyle and Control Metabolic Conditions of T2D. International Journal of Diabetes \& Its Complications 2(3): 1-7. Retrieved from http://cmepub.com/pdfs/ using-mathphysical-medicine-and-artificial-intelligencetechnology-to-manage-lifestyle-and-control-metabolicconditions-of-t2d-412.pdf

5. Hsu, Gerald C (2018) A Clinic Case of Using MathPhysical Medicine to Study the Probability of Having a Heart Attack or Stroke Based on Combination of Metabolic Conditions, Lifestyle, and Metabolism Index. Journal of Clinical Review \& Case Reports 3(5): 1-2. Retrieved from https://www.opastonline.com/wp-content/ uploads/2018/07/a-clinic-case-of-using-math-physicalmedicine-to-study-the-probability-of-having-a-heartattack-or-stroke-based-on-combination-of-metabolicconditions-lifestyle-and-metabolism-index-jcrc-2018.pdf
Copyright: (C2020 Gerald C. Hsu. This is an open-access article distributed under the terms of the Creative Commons Attribution License, which permits unrestricted use, distribution, and reproduction in any medium, provided the original author and source are credited. 